\title{
A System for Electrocardiographic Studies in the Community
}

\author{
Rene Ivan Gonzalez-Fernandez, Jorge Aguilera-Perez, Gisela Montes de Oca-Colina, Marisabel \\ Lopez-Fernandez, Pedro L. Gonzalez-Acosta and Miguel Portieles-Perez \\ ICID Digital Medical Technology, Havana, Cuba
}

\begin{abstract}
The proposed system is focused on the early detection and follows up of persons suffering cardiac disturbances in the community. The system is composed by a portable device for standard ECG recording, henceforth Recorder, and a desktop application, called Analyzer, to storage and study the signals acquired. The Recorder acquires and processes the standard ECG automatically, providing monitoring capabilities for emergency situations too. The patient data, the ECG and measurements are stored on the Recorder to be transmitted to the Analyzer later. The received information is stored in a database; the trend of several parameters associated with heart disease is computed for each patient. The studied parameters are: spatial dispersion of $Q T$ interval for malignant arrhythmias, the Selvester's Score for heart attacks, the Sokolow's index and Cornell's index for ventricular dilatation or hypertrophy. In an emergency situation, the ECG can be transmitted in real time to a Telecardiology system where experts analyze the incoming signal to indicate how to treat the patient. The same standard and the CSE database were used to test the ECG's processing algorithms. This approach seems a useful tool to detect cardiac disturbances in their early stages and reduce their mobility without significant costs.
\end{abstract}

\section{Introduction}

The annual statistics of the World Health Organization (WHO) show that heart disease is one of the three main causes of morbidity and mortality in the whole word [1]. Important economic resources have been invested in the development of sophisticated devices for the diagnosis and follow-up of heart diseases, but it is an accepted fact that the prevention and early detection of heart disease contributes significantly to minimizing the damage they cause. A strategy that combines appropriate technology with trained medical personnel and the dissemination of a healthy life style can have a very positive impact on the battle against these diseases.

Based on the above, a system has been developed for the study of cardiac diseases in the community with a minimum discomfort for people suffering this kind of disturbances. In this way, it is intended to detect the heart disease very early in people prone to suffer it, such as persons with high blood pressure, diabetics, smokers, etc. Also, the proposed system is intended to follow up the evolution of those patients who have already suffered a heart attack.

A Windows-based application and a small batterypowered twelve-lead electrocardiographic device compose the proposed system. The philosophy to follow is to enroll people to study in the proposed system and perform ECGs periodically in order to study the trend of several electrocardiographic parameters that characterize different cardiac disorders. The aim of this paper is to discuss the main features of the proposed system. Also, the main results of the technical tests made according to the IEC 60601-2-25 standard will be discussed.

\section{Materials and methods}

The proposed system is focused on the trend of several parameters to predict the occurrence of cardiac disturbances in apparently healthy persons or the beginning of complications in persons who had previously had heart attacks. The system is composed by a portable device for rest ECG acquisition, henceforth Recorder, and a Windows application, called Analyzer.

The Recorder was designed to be used in a wide range of places because it will be move out to the homes of the persons to be study. This device is based on an ARM9 microprocessor operating at a frequency of $400 \mathrm{MHz}$; also it has the following features:

- High resolution color-LCD with touch screen interface.

- Soft keypad for basic operations.

- Eight-channel ECG amplifier.

- USB interface.

- SD memory.

- Battery pack and medical grade's power supply.

The high resolution color-LCD is basic for a friendly interface with the operator. Graphics, digital signals, parameter values and text are combined to offer complete information about the studied patient based on his rest 
ECG. A six-key keypad associated to basic operations complement the user interface.

The ECG amplifier includes an analog band pass filter to limit the signal frequency spectrum between 0.05 and $100 \mathrm{~Hz}$, according to the IEC 60601-2-25 standard [2]. Circuits connected directly to the patient are protected against defibrillator discharge through $10 \mathrm{k} \Omega$ resistors and includes the classic right-leg circuit to improve the common mode rejection ratio [3]. The detection of pacemaker spikes is based on lead II analysis; the signal at the output of the instrumentation amplifier is passed through a filter that isolates the peaks and set an output digital pulse each time identifies one of these events. Furthermore, a trace recover circuit was implemented to recover the baseline level when amplifiers are satured because of disconnection of one or more electrodes.

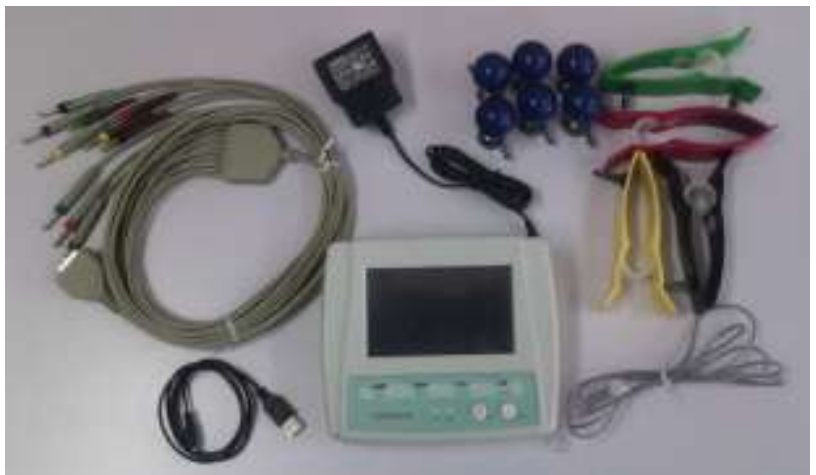

Figure 1. The Recorder and its accessories.

The eight independent leads (I, II, V1, V2, V3, V4, V5 and V6) are digitized simultaneously at a frequency of $500 \mathrm{~Hz}$ with a twelve-bit A / D converter; the LSB value is 3.15 microvolts. Classic expressions are used to compute the other four leads (III, aVR, aVL and aVF) because leads I and II were acquired simultaneously [4]. When the signal is digitized, a digital filtering scheme consists of a FIR filter of the moving average type (expression 1) and a $60 \mathrm{~Hz}$ notch filter is applied in real time to improve the signal quality.

$$
y(k)=\frac{1}{K^{2}} \sum_{m=k-K+1}^{k} \sum_{n=m-K+1}^{m} x(n)-\frac{1}{L^{2}} \sum_{m=k-L+1}^{k} \sum_{n=m-K+1}^{m} x(n)
$$

where:

$\mathrm{x}(\mathrm{n})$ : input signal

$\mathrm{y}(\mathrm{k})$ : output filtered signal

$\mathrm{K}$, L: filter constant

The QRS complexes are detected in real time to calculate the heart rate when ECG is acquired. An auxiliary function called Function of Spatial Velocity (FSV) is used to detect these complexes because this function allows to easily differentiating the areas associated with high frequency components [4]. Two thresholds are computed and updated to identify the QRS complexes and their onset and offset.

$$
F S V(k)=\sum_{i=1}^{C}[x(i, k)-x(i, k-1)]^{2}
$$

where:

$\mathrm{y}(\mathrm{k})$ : the FSV value

$\mathrm{x}(\mathrm{i}, \mathrm{k})$ : $\mathrm{ECG}$ for lead I at k time.

$\mathrm{C}$ : number of simultaneous leads

When a QRS complex is detected, its previous RR interval is measured and the mean value of this parameter is updated to compute the mean heart rate every ten seconds. The ECG is displayed on the format selected by the operator together with the heart rate value.

If the operator selects the option to store the signal, the acquisition process is stopped and the samples corresponding to the last ten seconds are analyzed automatically. The main events measured for each of the leads are the following:

- $\quad$ Amplitudes and durations of P, P', Q, R, S, R' and $\mathrm{T}$ waves.

- ST segment deviation at the beginning, the middle and the end of this event.

- Width of QRS complexes

- Duration of PR and QT intervals.

- Spatial dispersion of QT interval.

- Sokolow's index and Cornell's index.

- Selvester's Score.

- Ventricular Activation Time (VAT).

The algorithms to identify the electrocardiographic events are based on ECG spectral properties and heuristic procedures. Each digital ECG is store together with the automatic measurements and the patient's general data in a local Recorder's database to be transmitted to the Analyzer lately; a proprietary format ensures its confidentiality.

Each patient should be enrolled using the Analyzer; a friendly data entry is available for this purpose. Typically, an Analyzer and several Recorders work together, so the Analyzer will distribute the patients among the Recorders using a USB channel to transmit the general data of the patients.

An ECG from each patient will be captured at home periodically; physicians will set the frequency according to the characteristics of each patient and his pathology. All the ECG tests will be stored in the Analyzer's database, so it will grow as time goes by and physicians will be able to study the trend of several parameters strongly associated to dangerous cardiac diseases. The cardiac disturbances to study will be the following:

- Malignant arrhythmias: The trend of the QT interval dispersion will be used to predict this disturbance. Isolated values of this parameter are associated to dangerous conditions in several specialized publications $[5,6]$.

- Ventricular hypertrophy: Cornell and Sokolow indexes will be used to characterize the 
behaviour of this disease. These are classic index, but the proposed approach is new.

- Heart attack: Serial study of this score is proposed to evaluate the rehabilitation or deterioration of people who have suffered heart attacks.

The Analyser provides an easy to use graphic interface in order to help physicians in the data analysis. The information in grouped by patients and ordered according to the cardiac disturbance. A screen is dedicated to Hypertrophy study, other to Selvester's Score and so on.

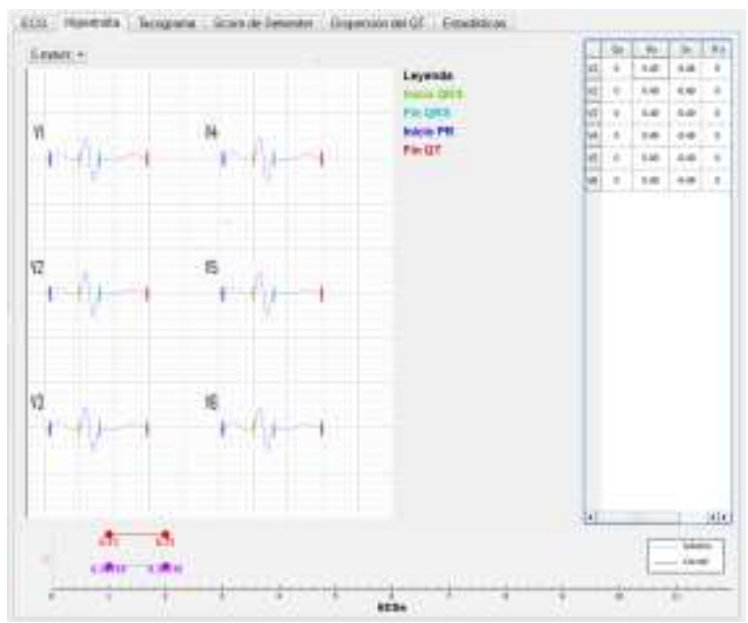

Figure 2. The screen of Hypertrophy Analysis.

The Analyzer's database is the heart of the proposed solution because it contains the information necessary to predict if any studied patient will be in a good cardiovascular condition or if he will be in a dangerous condition.

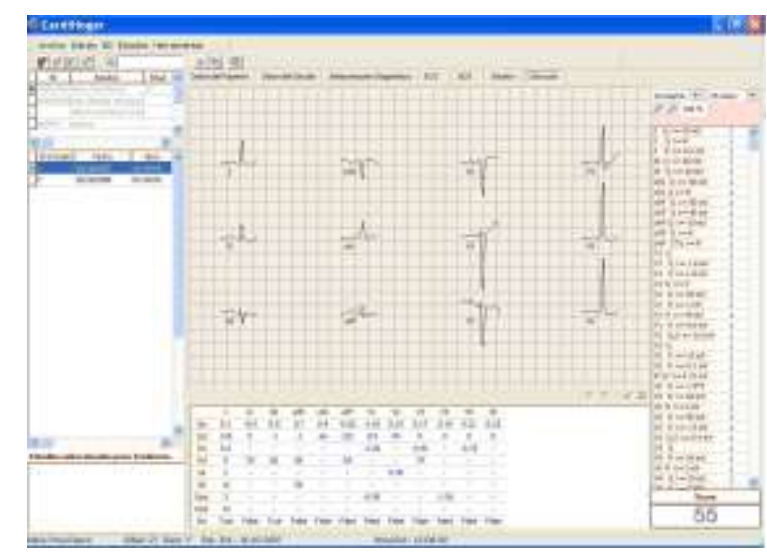

Figure 3. The screen of Selvester's Score.

The Analyzer software was developed in $\mathrm{C}++$ language using Qt Creator platform. A Linux embedded distribution was used as the Recorder's operating system. Also, the software of the Recorder was developed using the same programming tools to improve the productivity sharing several technical solutions. The principles of the Object Oriented Programming paradigm were applied.

\section{Results and discussion}

The global performance of the proposed system was tested with fifteen voluntaries and two experimented physicians. More than two-hundred ECGs have been acquired and stored on the system database to evaluate all the features of the proposed system. Also, error conditions were provoked to evaluate the system response.

Five prototypes of the Recorder were manufactured and tested successfully. Three devices were dedicated to technical tests and two devices were used to capture ECGs from voluntaries and simulators. Also, a first version of the Analyzer was completed. The IEC 606012-25 was the reference for parametric and electrical safety tests because this document set the requirements for the basic safety and essential performance of electrocardiographic devices. Some of the main results are shown in Table 1; the results of all tests were always within the allowable limits.

Table 1. Main results in IEC 60601-2-25 tests.

\begin{tabular}{ll}
\hline Parameter & Result \\
\hline Frequency response & 0.05 to $100 \mathrm{~Hz}$ \\
Input dynamic range & $\pm 5 \mathrm{mV}$ \\
Accuracy of the sensitivity & Less than $\pm 5 \%$ \\
ECG channel interference & $\begin{array}{l}2 \% \text { of applied } \\
\text { signal }\end{array}$ \\
Intrinsic channel noise & Less than $30 \mu \mathrm{V}$ \\
Overload tolerance & $1 \mathrm{Vp-p}$ \\
Common Mode Rejection Ratio & Greater than 90 \\
& dB \\
Patient auxiliary current & Less than $50 \mu \mathrm{A}$ \\
Permanent leakage current & Less than $300 \mu \mathrm{A}$ \\
\hline
\end{tabular}

The results have a full correspondence with the IEC 60601-2-25 specifications; so the safety of the electronic design solutions proposed have been demonstrated. Also, the state of the art of the proposed solution is appropriated.

The algorithms developed for ECG processing were tested with CSE and CTS databases according to the IECG 60601-2-25.

All the QRS complexes of the ECGs used to test the algorithm were detected; these signals are included on the CTS and CSE databases. There were no false positives or false negatives.

The accuracy of the automatic amplitude measurements within the QRS complex was analyzed according to the IEC 60601-2-25 standard; it was found that no measurements deviated from the reference more than 25 microvolts for amplitudes little than 500 microvolts or more than $5 \%$ for amplitudes greater than 500 microvolts. This behaviour is established by the IEC standard as appropriate. The $\mathrm{P}$ wave analysis was 
included in Table 2 and the performance was similar to the results for the PQ interval as expected.

Table 2. Automatic measurements on analytical ECGs.

\begin{tabular}{lcc}
\hline Interval & $\begin{array}{c}\text { Mean } \\
\text { error } \\
(\mathrm{ms})\end{array}$ & $\begin{array}{c}\text { Standard } \\
\text { Deviation } \\
(\mathrm{ms})\end{array}$ \\
\hline P wave & 7.02 & 6.48 \\
PQ interval & 6.81 & 6.77 \\
QRS complex & 4.10 & 5.40 \\
QT interval & 7.88 & 8.84 \\
\hline
\end{tabular}

The whole system has also been preliminarily tested with simulated signals and volunteers. Ten men and five women were voluntaries to test the proposed system; their age was between 25 and 62 years. All of them are healthy people. ECGs were captured at the institution where the authors work with the consent of the volunteers. Two persons without experience in rest ECG were trained to use the Recorder; they declared the Recorder was easy to use for them at the end of the tests.

The following errors were provoked during the system operation:

- Electrode disconnection from the patient: The Recorder always detected properly the failing contact electrodes. This information was displayed on the Recorder's screen to alert the operator.

- Patient cable disconnection from the Recorder: The device always detected this condition and displayed the corresponding message to alert the operator.

- Wrong data during the patient registration: The data entry process is strongly checked. When the operator tries to enter a wrong data, a message is displayed and the wrong data is rejected.

- Sudden power down during ECG acquisition: The Recorder keeps working with batteries when power fails.

- Accidental device power off: The Recorder always requests an operator confirmation before executing the shutdown. It is impossible to accidentally execute this action.

- Mechanical damages: The Recorder was dropped from a meter high and its operation was not interrupted due to impact.

The global performance of the proposed system was robust and according to the goals of the research project. The operation of the Recorder and the Analyzer has been stable and there were not devices out of order or software's malfunctions. However, a more extensive test is mandatory for definitive criteria.

\section{Conclusions}

The proposed system has been completed with satisfactory results. All the IEC tests have been passed successfully; the electronic and mechanical solutions implemented in the Recorder are safety for patients and operators. The same can be said of software.

The Analyzer was considered as a tool easy to use and powerful to manage and to interpret the ECG data collected. It worked stably and never a catastrophic error occurs during the tests. The graphical tools make easy the study of cases processed by the system. The proposed system seems a useful tool for the study of heart disease at the community level.

\section{Acknowledgements}

The authors of this paper would like to acknowledge the medical advice provided by cardiologists at the Institute of Cardiology and the "Hermanos Ameijeiras" Hospital, both in Havana, Cuba.

\section{References}

[1] WHO. Guidelines for primary health care in low-resource settings. WHO Library Cataloguing-in-Publication Data. ISBN 978924154839 7, 2012.

[2] IEC 60601-2-25 Medical electrical equipment. Part 2-25: Particular requirements for the basic safety and essential performance of electrocardiographs. Edition 2.0, 2011-10.

[3] Bronzino J D, Peterson D R. The Biomedical Engineering Handbook. CRC Press. ISBN 9781439825334. 2015.

[4] Macfarlane P, Macfarlane D K, Podolski M. Mingocare: Un Nuevo programa para la interpretación electrocardiográfica asistida por computador, Electromédica 1984; 52(4).

[5] Lopes N H et al. QT Interval Dispersion Analysis in Acute Myocardial Infarction Patients: Coronary Reperfusion Effect. Arquivos Brasileiros de Cardiología, 2006; 87(2):116-123.

[6] Valladares F, Álvarez F, Iraola M, Ibargollín R. Valor del sistema de puntuación del QRS-Selvester en el infarto miocárdico agudo, Revista Cubana de Cardiología y Cirugía Cardiovascular 2000; 14(2):94-98.

Address for correspondence.

Rene Ivan Gonzalez Fernandez.

Calle 202 \#1704 entre 17 y 19, reparto Siboney, municipio Playa. La Habana, Cuba.

rigf58@yahoo.com

rigf58@gmail.com 